SELECCIONES MATEMÁTICAS
Universidad Nacional de Trujillo
ISSN: 2411-1783 (Online)
2020; Vol. 7(1): 25-28.

Uniquely list colorability of the graph $K_{2}^{n}+O_{m}$ Le Xuan Hung ${ }^{\circledR}$

Received, Jan. 28, 2020

Accepted, Jun. 01, 2020

How to cite this article:

Hung L.X. Uniquely list colorability of the graph $K_{2}^{n}+O_{m}$. Selecciones Matemáticas. 2020;7(1):25-28. http: //dx.doi.org/10.17268/sel.mat.2020.01.03

\begin{abstract}
Given a list $L(v)$ for each vertex $v$, we say that the graph $G$ is $L$-colorable if there is a proper vertex coloring of $G$ where each vertex $v$ takes its color from $L(v)$. The graph is uniquely $k$-list colorable if there is a list assignment $L$ such that $|L(v)|=k$ for every vertex $v$ and the graph has exactly one $L$-coloring with these lists. In this paper, we characterize uniquely list colorability of the graph $G=K_{2}^{n}+O_{m}$. We shall prove that if $n=2$ then $G$ is uniquely 3-list colorable if and only if $m \geq 9$, if $n=3$ and $m \geq 1$ then $G$ is uniquely 3-list colorable, if $n \geq 4$ then $G$ is uniquely $k$-list colorable with $k=\left\lfloor\frac{m}{2}\right\rfloor+1$ and if $m \geq n-1$ then $G=K_{2}^{n}+O_{m}$ is UnLC.
\end{abstract}

Keywords . Vertex coloring (coloring), list coloring, uniquely list colorable graph, complete r-partite graph.

1. Introduction. All graphs considered in this paper are finite undirected graphs without loops or multiple edges. If $G$ is a graph, then $V(G)$ and $E(G)$ (or $V$ and $E$ in short) will denote its vertex-set and its edge-set, respectively. The set of all neighbours of a subset $S \subseteq V(G)$ is denoted by $N_{G}(S)$ (or $N(S)$ in short). Further, for $W \subseteq V(G)$ the set $W \cap N_{G}(S)$ is denoted by $N_{W}(S)$. If $S=\{v\}$, then $N(S)$ and $N_{W}(S)$ are denoted shortly by $N(v)$ and $N_{W}(v)$, respectively. For a vertex $v \in V(G)$, the degree of $v$ (resp., the degree of $v$ with respect to $W$ ), denoted by $\operatorname{deg}(v)\left(\right.$ resp., $\operatorname{deg}_{W}(v)$ ), is $\left|N_{G}(v)\right|\left(\right.$ resp., $\left|N_{W}(v)\right|$ ). The subgraph of $G$ induced by $W \subseteq V(G)$ is denoted by $G[W]$. The empty and complete graphs of order $n$ are denoted by $O_{n}$ and $K_{n}$, respectively. Unless otherwise indicated, our graph-theoretic terminology will follow [2].

A graph $G=(V, E)$ is called $r$-partite graph if $V$ admits a partition into $r$ classes $V=V_{1} \cup V_{2} \cup \ldots \cup V_{r}$ such that the subgraphs of $G$ induced by $V_{i}, i=1, \ldots, r$, is empty. An $r$-partite graph in which every two vertices from different partition classes are adjacent is called complete $r$-partite graph and is denoted by $K_{\left|V_{1}\right|,\left|V_{2}\right|, \ldots,\left|V_{r}\right|}$. The complete $r$-partite graph $K_{\left|V_{1}\right|,\left|V_{2}\right|, \ldots,\left|V_{r}\right|}$ with $\left|V_{1}\right|=\left|V_{2}\right|=\ldots=\left|V_{r}\right|=s$ is denoted by $K_{s}^{r}$

Let $G_{1}=\left(V_{1}, E_{1}\right), G_{2}=\left(V_{2}, E_{2}\right)$ be two graphs such that $V_{1} \cap V_{2}=\emptyset$. Their union $G=G_{1} \cup G_{2}$ has, as expected, $V(G)=V_{1} \cup V_{2}$ and $E(G)=E_{1} \cup E_{2}$. Their join defined is denoted $G_{1}+G_{2}$ and consists of $G_{1} \cup G_{2}$ and all edges joining $V_{1}$ with $V_{2}$.

Let $G=(V, E)$ be a graph and $\lambda$ is a positive integer.

A $\lambda$-coloring of $G$ is a mapping $f: V(G) \rightarrow\{1,2, \ldots, \lambda\}$ such that $f(u) \neq f(v)$ for any adjacent vertices $u, v \in V(G)$. The smallest positive integer $\lambda$ such that $G$ has a $\lambda$-coloring is called the chromatic number of $G$ and is denoted by $\chi(G)$. We say that a graph $G$ is $n$-chromatic if $n=\chi(G)$.

Let $(L(v))_{v \in V}$ be a family of sets. We call a coloring $f$ of $G$ with $f(v) \in L(v)$ for all $v \in V$ is a list coloring from the lists $L(v)$. We will refer to such a coloring as an $L$-coloring. The graph $G$ is called $\lambda$-list-colorable, or $\lambda$-choosable, if for every family $(L(v))_{v \in V}$ with $|L(v)|=\lambda$ for all $v$, there is a coloring of $G$ from the lists $L(v)$. The smallest positive integer $\lambda$ such that $G$ has a $\lambda$-choosable is called the listchromatic number, or choice number of $G$ and is denoted by $\operatorname{ch}(G)$. The idea of list colorings of graphs is due independently to V. G. Vizing [14] and to P. Erdös, A. L. Rubin, and H. Taylor [7].

${ }^{*}$ HaNoi University for Natural Resources and Environment 41 A, Phu Dien Road, Phu Dien precinct, North Tu Liem district, Hanoi, Vietnam (lxhung@hunre.edu.vn). 
Let $G$ be a graph with $n$ vertices and suppose that for each vertex $v$ in $G$, there exists a list of $k$ colors $L(v)$, such that there exists a unique $L$-coloring for $G$, then $G$ is called a uniquely $k$-list colorable graph or a UkLC graph for short. The idea of uniquely colorable graph was introduced independently by Dinitz and Martin [6] and by Mahmoodian and Mahdian [10].

The list coloring model can be used in the channel assignment. The fixed channel allocation scheme leads to low channel utilization across the whole channel. It requires a more effective channel assignment and management policy, which allows unused parts of channel to become available temporarily for other usages so that the scarcity of the channel can be largely mitigated [15]. It is a discrete optimization problem. A model for channel availability observed by the secondary users is introduced in [15]. The research of list coloring consists of two parts: the choosability and the unique list colorability. In [9], we characterized list-chromatic number of the graph $G=K_{2}^{n}+O_{m}$.

In this paper, we characterize uniquely list colorability of the graph $G=K_{2}^{n}+O_{m}$. We shall prove that if $n=2$ then $G$ is uniquely 3 -list colorable if and only if $m \geq 9$, if $n=3$ and $m \geq 1$ then $G$ is uniquely 3-list colorable, if $n \geq 4$ and $m \geq 1$ then $G$ is uniquely $k$-list colorable with $k=\left\lfloor\frac{n}{2}\right\rfloor+1$ and if $m \geq n-1$ then $G=K_{2}^{n}+O_{m}$ is $\mathrm{U} n \mathrm{LC}$.

2. Preliminaries. If a graph $G$ is not uniquely $k$-list colorable, we also say that $G$ has property $M(k)$. So $G$ has the property $M(k)$ if and only if for any collection of lists assigned to its vertices, each of size $k$, either there is no list coloring for $G$ or there exist at least two list colorings. The least integer $k$ such that $G$ has the property $M(k)$ is called the m-number of $G$, denoted by $m(G)$. This conception was originally introduced by Mahmoodian and Mahdian in [10].

Example. Set the graph $G=(V, E)$ with $V=\left\{v_{1}, v_{2}, v_{3}, v_{4}\right\}$ and $E=\left\{v_{1} v_{3}, v_{1} v_{4}, v_{2} v_{3}, v_{2} v_{4}, v_{3} v_{4}\right\}$. It is not difficult to see that $G$ is $\mathrm{U} 2 \mathrm{LC}, G$ has the property $M(3)$ and $m(G)=3$

We need the following Lemmas 1-8 to prove our results.

Lemma 1 ([10]). A connected graph $G$ has the property $M(2)$ if and only if every block of $G$ is either a cycle, a complete graph, or a complete bipartite graph.

If $n=1$ then $G=K_{2}^{n}+O_{m}$ is a complete bipartite graph, by Lemma 1, $G$ has the property $M(2)$.

Lemma 2 ([10]). For every graph $G$ we have $m(G) \leq \leq|E(\bar{G})|+2$.

Lemma 3. If $G=K_{2}^{n}+O_{m}$ is UkLC then $k \leq 2 n+1$.

Proof: Suppose that $G$ is UkLC. Then there exists a list of $k$ colors $L(v)$, such that there exists a unique $L$-coloring $f$ for $G$. Let $V(G)=V_{1} \cup V_{2} \cup \ldots \cup V_{n+1}$ is a partition of $V(G)$ such that $\left|V_{1}\right|=\left|V_{2}\right|=\ldots=$ $\left|V_{n}\right|=2,\left|V_{n+1}\right|=m$ and for every $i=1,2, \ldots, n+1$ the subgraphs of $G$ induced by $V_{i}$, is empty graph. Set $V_{n+1}=\left\{v_{1}, v_{2}, \ldots, v_{m}\right\}$.

It is clear that $\left|f\left(V_{1} \cup V_{2} \cup \ldots \cup V_{n}\right)\right| \leq 2 n$ and $\left|L\left(v_{1}\right) \backslash\left\{f\left(v_{1}\right)\right\}\right|=k-1$. If $k>2 n+1$ then $\left|L\left(v_{1}\right) \backslash\left\{f\left(v_{1}\right)\right\}\right|>2 n$. So there exists $c \in L\left(v_{1}\right) \backslash\left\{f\left(v_{1}\right)\right\}$ such that $c \notin f\left(V_{1} \cup V_{2} \cup \ldots \cup V_{n}\right)$. It follows that there exists a unique $L$-coloring $f^{\prime}$ for $G: f(v)=f^{\prime}(v)$ if $v \in V(G) \backslash\left\{v_{1}\right\}$ and $f\left(v_{1}\right)=c$. It is not difficult to see that $f \neq f^{\prime}$, a contradiction. Thus, $k \leq 2 n+1$.

Lemma 4 ([10]). Every UkLC graph has at least $3 k-2$ vertices.

Lemma 5. If $G=K_{2}^{n}+O_{m}$ is a graph with $n=2$ and $1 \leq m \leq 2$ then $m(G)=3$.

Proof: By Lemma 1, $G$ is U2LC. Suppose that $G$ is U3LC. By Lemma 4, $|V(G)| \geq 7$, a contradiction. So $m(G)=3$.

Lemma 6 ([10]). If $G=K_{2}^{n}+O_{m}$ is a graph with $n=2$ and $m=3$ then $m(G)=3$.

Lemma 7 ([17]). If $G=K_{2}^{n}+O_{m}$ is a graph with $n=2$ and $4 \leq m \leq 8$ then $m(G)=3$.

Lemma 8. If $G=K_{2}^{n}+O_{m}$ is a graph with $n=2$ and $1 \leq m \leq 8$ then $m(G)=3$.

Proof: It follows from Lemma 5, Lemma 6 and Lemma 7.

Lemma 9 ([17]). If $n=2$ and $m=9$ then $G=K_{2}^{n}+O_{m}$ is $U 3 L C$.

3. Main Results. Set the graph $G=K_{2}^{n}+O_{m}$. Let $V(G)=V_{1} \cup V_{2} \cup \ldots \cup V_{n+1}$ is a partition of $V(G)$ such that $\left|V_{1}\right|=\left|V_{2}\right|=\ldots=\left|V_{n}\right|=2,\left|V_{n+1}\right|=m$ and for every $i=1,2, \ldots, n+1$ the subgraphs of $G$ induced by $V_{i}$, is empty graph.

Theorem 1. If $n=2$ then $G=K_{2}^{n}+O_{m}$ is $U 3 L C$ if and only if $m \geq 9$.

Proof: First we prove the necessity. Suppose that $G=K_{2}^{2}+O_{m}$ is U3LC. If $1 \leq m \leq 8$ then by Lemma $8, m(G)=3$, a contradiction. Therefore, $m \geq 9$.

Now we prove the sufficiency. We prove $G=\overline{K_{2}^{2}}+O_{m}$ is U3LC by induction on $m$. If $m=9$ then by Lemma 9, $G$ is U3LC. So let $m>9$ and assume the assertion for smaller values of $m$. 
Set $V_{3}=\left\{v_{1}, v_{2}, \ldots, v_{m}\right\}$ and $G^{\prime}=G-v_{m}$. It is clear that $G^{\prime}=K_{2}^{2}+O_{m-1}$. By the induction hypothesis, for each vertex $v$ in $G^{\prime}$, there exists a list of 3 colors $L_{v}^{\prime}$, such that there exists a unique coloring $f^{\prime}$ for $G^{\prime}$.

We assign the following lists for the vertices of $G: L(v)=L^{\prime}(v)$ if $v \in V\left(G^{\prime}\right), L\left(v_{m}\right)=L^{\prime}\left(v_{m-1}\right)$.

A unique coloring $f$ of $G$ exists from the assigned lists: $f(v)=f^{\prime}(v)$ if $v \in V\left(G^{\prime}\right), f\left(v_{m}\right)=$ $f^{\prime}\left(v_{m-1}\right)$.

Theorem 2. If $n=3$ and $m \geq 1$ then $G=K_{2}^{n}+O_{m}$ is $U 3 L C$.

Proof: Set $V_{1}=\left\{u_{1}, u_{2}\right\}, V_{2}=\left\{u_{3}, u_{4}\right\}, V_{3}=\left\{u_{5}, u_{6}\right\}$ and $V_{4}=\left\{v_{1}, v_{2}, \ldots, v_{m}\right\}$.

We assign the following lists for the vertices of $G: L\left(u_{1}\right)=\{1,4,5\}, L\left(u_{2}\right)=\{2,4,5\}, L\left(u_{3}\right)=$ $\{1,2,3\}, L\left(u_{4}\right)=\{3,4,5\}, L\left(u_{5}\right)=\{1,2,4\}, L\left(u_{6}\right)=\{3,4,5\}, L\left(v_{1}\right)=L\left(v_{2}\right)=\ldots=L\left(v_{m}\right)=$ $\{3,4,5\}$.

A unique coloring $f$ of $G$ exists from the assigned lists: $f\left(u_{1}\right)=1, f\left(u_{2}\right)=2, f\left(u_{3}\right)=3, f\left(u_{4}\right)=3$, $f\left(u_{5}\right)=4, f\left(u_{6}\right)=4, f\left(v_{1}\right)=f\left(v_{2}\right)=\ldots=f\left(v_{m}\right)=5$.

Theorem 3. If $n \geq 4$ and $m \geq 1$ then $G=K_{2}^{n}+O_{m}$ is UkLC with $k=\left\lfloor\frac{n}{2}\right\rfloor+1$.

Proof: Set $V_{i}=\left\{u_{i 1}, u_{i 2}\right\}$ for every $i=1,2, \ldots, n$ and $V_{n+1}=\left\{v_{1}, v_{2}, \ldots, v_{m}\right\}$. Put $t=\left\lfloor\frac{n}{2}\right\rfloor$. We assign the following lists for the vertices of $G$ :

$L\left(u_{i 1}\right)=\{1,2, \ldots, t+1\}$ for every $i=1,2, \ldots, t+1 ;$

$L\left(u_{i 2}\right)=\{t+2, t+3, \ldots, 2 t+1, i\}$ for every $i=1,2, \ldots, t+1$;

$L\left(u_{(t+i) j}\right)=\{2,3, \ldots, t+1, t+1+i\}$ for every $i=1,2, \ldots, n-t-1, j=1,2$;

$L\left(v_{1}\right)=L\left(v_{2}\right)=\ldots=L\left(v_{m}\right)=\{2,3, \ldots, t+1, n+1\}$.

A unique coloring $f$ of $G$ exists from the assigned lists:

$f\left(u_{i 1}\right)=i$ for every $i=1,2, \ldots, t+1$;

$f\left(u_{i 2}\right)=i$ for every $i=1,2, \ldots, t+1$;

$f\left(u_{(t+i) j}\right)=t+1+i$ for every $i=1,2, \ldots, n-t-1, j=1,2$;

$f\left(v_{1}\right)=f\left(v_{2}\right)=\ldots=f\left(v_{m}\right)=n+1$.

Corollary 1. If $G=K_{2}^{n}+O_{m}$ is a graph with $n=4$ and $m=1$ then $m(G)=4$.

Proof: By Theorem 3, $G$ is U3LC. If $G$ is U4LC then by Lemma $4,|V(G)| \geq 10$, a contradiction. So $m(G)=4$.

Now we characterize uniquely list colorability of the graph $G=K_{2}^{n}+O_{m}$ with $m \geq n-1$.

\section{Theorem 4.}

If $m \geq n-1$ then $G=K_{2}^{n}+O_{m}$ is $U n L C$.

Proof:

Set $V_{i}=\left\{u_{i 1}, u_{i 2}\right\}$ for every $i=1,2, \ldots, n$ and $V_{n+1}=\left\{v_{1}, v_{2}, \ldots, v_{m}\right\}$. We assign the following lists for the vertices of $G$ :

$L\left(u_{i 1}\right)=\{1,2, \ldots, n\}$ for every $i=1,2, \ldots, n$;

$L\left(u_{i 2}\right)=\{n+1, n+2, \ldots, 2 n-1, i\}$ for every $i=1,2, \ldots, n$;

$L\left(v_{j}\right)=\{1,2, \ldots, n-1, n+j\}$ for every $j=1,2, \ldots, n-1$;

$L\left(v_{j}\right)=L\left(v_{n-1}\right)$ for every $j=n, n+1, \ldots, m$.

A unique coloring $f$ of $G$ exists from the assigned lists:

$f\left(u_{i 1}\right)=i$ for every $i=1,2, \ldots, n$;

$f\left(u_{i 2}\right)=i$ for every $i=1,2, \ldots, n$;

$f\left(v_{j}\right)=n+j$ for every $j=1,2, \ldots, n-1$;

$f\left(v_{j}\right)=f\left(v_{n-1}\right)$ for every $j=n, n+1, \ldots, m$.

Finally we determine $m(G)$ number for the graph $G=K_{2}^{n}+O_{m}$ with $n-1 \leq m \leq n$.

Corollary 2. If $G=K_{2}^{n}+O_{m}$ is a graph with $n-1 \leq m \leq n$ then $m(G)=n+1$.

Proof: By Theorem 4, $G$ is UnLC. If $G$ is $\mathrm{U}(n+1) \mathrm{LC}$ then by Lemma $4,|V(G)| \geq 3 n+1$, a contradiction. So $m(G)=n+1$.

ORCID and License

Le Xuan Hung https://orcid.org/0000-0003-4560-2892

This work is licensed under the Creative Commons Attribution-NoComercial-ShareAlike 4.0. 


\section{References}

[1] Behzad M. Graphs and thei chromatic number. Doctoral Thesis (Michigan State University), 1965.

[2] Behzad M, Chartrand G. Introduction to the theory of graphs. Allyn and Bacon, Boston, 1971.

[3] Behzad M, Chartrand G, Cooper J. The coloring numbers of complete graphs. J. London Math. Soc. 1967; 42:226-228.

[4] Bondy J, Murty U. Graph theory with applications. MacMillan, 1976.

[5] Diestel R. Graph Theory, Springer - Verlag New Your, 2000.

[6] Dinitz J. Martin W. The stipulation polynomial of a uniquely list colorable graph. Austran. J. Combin. 1995; 11:105-115.

[7] Erdös P, Rubin A, Taylor H. (1979) Choosability in graphs. In Proceedings of west coast conference on combinatorics, graph theory, and computing, number 26 in Congr. Numer. pages 125-157, Arcata, CA, September.

[8] Ghebleh M, Mahmoodian E. On uniquely list colorable graphs. Ars Combin. 2001; 59:307-318.

[9] Hung L. List-chromatic number and chromatically unique of the graph $K_{2}^{r}+O_{k}$. Selecciones Matemáticas, Universidad Nacional de Trujillo. 2019; 06(01):26-30.

[10] Mahdian M, Mahmoodian E. A characterization of uniquely 2-list colorable graphs. Ars Combin. 1999; 51:295-305.

[11] Read R. (1968) An introduction to chromatic polynomials. J. Combin. Theory. 1968; 4:52-71.

[12] Tan N, Hung L. On colorings of split graphs. Acta Mathematica Vietnammica. 2006; 31(3):195-204.

[13] Vizing V. On an estimate of the chromatic class of a p-graph. Discret. Analiz. 1964; 3:23-30.

[14] Vizing V. Coloring the vertices of a graph in prescribed colors. In Diskret. Analiz, number 29 in Metody Diskret. Anal. v Teorii Kodov i Shem. 1976; 3-10.

[15] Wang W, Liu X. List-coloring based channel allocation for open-spectrum wireless networks. In Proceedings of the IEEE International Conference on Vehicular Technology (VTC '05). 2005; 690-694.

[16] Wilson R. Introduction to graph theory. Longman group ltd, London, 1975.

[17] Zhao Y, Shan E. On characterization of uniquely 3-list colorable complete multipartite graphs. Discussiones Mathematicae Graph Theory. 2010; 30:105-114. 